\title{
ON THE COVERING AND THE ADDITIVITY NUMBER OF THE REAL LINE
}

\author{
KYRIAKOS KEREMEDIS
}

(Communicated by Andreas R. Blass)

\begin{abstract}
We show that the real line $R$ cannot be covered by $k$ many nowhere dense sets iff whenever $D=\left\{D_{i}: i \in k\right\}$ is a family of dense open sets of $R$ there exists a countable dense set $G$ of $R$ such that $\left|G \backslash D_{i}\right|<\omega$ for all $i \in k$. We also show that the union of $k$ meagre sets of the real line is a meagre set iff for every family $D=\left\{D_{i}: i \in k\right\}$ of dense open sets of $R$ and for every countable dense set $G$ of $R$ there exists a dense set $Q \subseteq G$ such that $\left|Q \backslash D_{i}\right|<\omega$ for all $i \in k$.
\end{abstract}

\section{NOTATION AND TERMINOLOGY}

The notation and terminology which we will use is standard and can be found in [8] or [9]. In particular, if $A, B$ are sets and $\lambda$ any cardinal finite or infinite, then $[A]^{\lambda},[A]^{<\lambda}$, and $[A] \leq \lambda$ denote the sets of all subsets of $A$ of size $\lambda,<\lambda$, and $\leq \lambda$ respectively. $\omega^{\omega}$ denotes the Baire space, i.e., the set of all functions ${ }^{\omega} \omega$ together with the topology, having as a base the collection of all (clopen) sets of the form

$$
[p]=\left\{f \in{ }^{\omega} \omega: p \subseteq f\right\}, \quad p \in \omega^{<\omega}=\bigcup\left\{{ }^{n} \omega: n \in \omega\right\} .
$$

We say that the family $A \subseteq[\omega]^{\omega}$ has the countable $\pi$-base property (c $\pi \mathrm{bp}$ ), iff there exists a $B \in\left[[\omega]^{\omega}\right]^{\omega}$, call it a $\pi$-base, such that

$$
(\forall a \in A)(\forall b \in B)(\exists d \in B)(d \subseteq a \cap b) .
$$

In order to avoid confusion, let us remark that the notions of $\mathrm{c} \pi \mathrm{bp}$ and a filter $A$ of $[\omega]^{\omega}$ having a countable base are not the same. $B$ need not be included in $A$. In fact, $B$ may contain disjoint sets.

$A \subseteq[\omega]^{\omega}$ has the strong finite intersection property (sfip), iff $|\cap Q|=\omega$ for every $Q \in[A]^{<\omega}$.

We say that a set $S \in[\omega]^{\omega}$ is an infinite pseudointersection of the family $A \subseteq[\omega]^{\omega}$ iff $(\forall a \in A)(|S \backslash a|<\omega)$.

Received by the editors April 1, 1993 and, in revised form, September 10, 1993; presented to the 3rd Greek Conference on Mathematical Analysis held in Ioannina, May 28-29, 1993.

1991 Mathematics Subject Classification. Primary 03E35; Secondary 03E40.

Key words and phrases. Covering number, additivity number, nowhere dense, meagre, bounding number, dominating number. 
Let $k, m$ be any infinite cardinal numbers less than the power of the continuum $\mathfrak{c}$. We define the following combinatorial statements:

$\operatorname{PS}(k) \equiv$ Every $A \in\left[[\omega]^{\omega}\right]^{\leq k}$ with the c $\pi$ bp has an infinite pseudointersection.

We remark here that it is consistent with ZFC that there exist sets $A \subseteq[\omega]^{\omega}$ having the sfip but no countable $\pi$-base (there are filters having no countable base). Indeed, let $(M, \epsilon)$ be a model of ZFC such that $M \vDash \mathfrak{p}=\omega_{1}+\operatorname{cov}(R)=$ $\omega_{2}$ (see [9, Theorem 3]), where $\mathfrak{p}$ is the pseudointersection number and $\operatorname{cov}(R)$ the covering number of the real line. Then there exists a set $A \subseteq[\omega]^{\omega},|A|=$ $\omega_{1}$, having the sfip but no infinite pseudointersection. If $B=\left\{b_{n}: n \in \omega\right\} \subseteq$ $[\omega]^{\omega}$ were a $\pi$-base for $A$, then, using $\operatorname{cov}(R)>\omega_{1}$, one can easily construct a pseudointersection $S$ for $A$ which is a contradiction.

$\operatorname{SPS}(k) \equiv$ For every $A \in\left[[\omega]^{\omega}\right]^{\leq k}$ and for every $\pi$-base $B \in\left[[\omega]^{\omega}\right]^{\omega}$ for $A$ there exists an infinite pseudointersection $S$ of $A$ meeting each member of $B$ is an infinite set.

$\operatorname{Equal}(k) \equiv\left(\forall F \in\left[{ }^{\omega} \omega\right]^{\leq k}\right)\left(\exists h \in^{\omega} \omega\right)(\forall f \in F)\left(\exists^{\infty} n\right)(f(n)=h(n))$, where $\exists^{\infty} n$ abbreviates the statement "there are infinitely many".

$\operatorname{Equal}^{*}(k) \equiv\left(\forall F \in\left[{ }^{\omega} \omega\right]^{\leq k}\right)\left(\exists h_{n} \in^{\omega} \omega\right)(\forall f \in F)\left(\forall^{\infty} n\right)(\exists m \in \omega)(f(n)=$ $\left.h_{n}(n)\right)$, where $\forall^{\infty} n$ abbreviates the statement "for all but finitely many".

Bounded $(k) \equiv\left(\forall F \in\left[{ }^{\omega} \omega\right] \leq k\right)\left(\exists h \in^{\omega} \omega\right)(\forall f \in F)\left(\forall^{\infty} n\right)(f(n) \leq h(n))$.

Weak Bounded $(k) \equiv\left(\forall F \in\left[{ }^{\omega} \omega\right]^{\leq k}\right)\left(\exists h \in^{\omega} \omega\right)(\forall f \in F)\left(\exists^{\infty} n\right)(f(n)<h(n))$.

The bounding number $\mathfrak{b}$ and the dominating number $\mathfrak{d}$ are the least cardinal numbers $k$ for which the statements $\operatorname{Bounded}(k)$ and Weak Bounded $(k)$ fail respectively.

Let $(X, T)$ be a topological space. A dense subset $Q$ of the poset $(T \backslash(\varnothing)$, $\subseteq)$ is called a $\pi$-base for $X$.

A set $N \subseteq X$ is called nowhere dense iff $\operatorname{int}(\bar{N})=\varnothing$, and a set $A \subseteq X$ is called meagre iff $A$ is the union of countable many nowhere dense sets. The covering number, $\operatorname{cov}(X)$, and the additivity number, $\operatorname{add}(X)$, of the space $X$ are given by:

$\operatorname{cov}(X) \equiv \min \{|D|: D$ is a family of dense open sets in $X$ with $\bigcap D=\varnothing\}$, $\operatorname{add}(X) \equiv \min \{|D|: D$ is a family of meagre sets of $X$ but $\cup D$ is not a meagre set of $X$ \} respectively.

All undefined terms are used as in $[5,8,10]$.

\section{INTRODUCTION AND PRELIMINARY RESULTS}

The covering number, $\operatorname{cov}(R)$, of the real line may be a singular cardinal number [11]. In this case, it has been shown by A. W. Miller in [13] that $\operatorname{cov}(R)$ has uncountable cofinality, and T. Bartoszynski and J. I. Ihoda have shown in [3] that its cofinality is greater than or equal to $\operatorname{add}(\mathbf{L})$, where $\operatorname{add}(\mathbf{L})$ is the additivity number of the ideal $\mathbf{L}$ of all Lebesque null sets of the real line. It is an open question (see $[3,4,15])$ whether $\operatorname{cf}(\operatorname{cov}(R))$ can be less than $\operatorname{add}(R)$. Note that if $\operatorname{cov}(R) \leq \mathfrak{b}$, then by Lemma 3 we have $\operatorname{cov}(R)=\operatorname{add}(R)$ and that if $\operatorname{cov}(R)=\mathfrak{d}$, then $\operatorname{cf}(\mathfrak{d}) \geq \mathfrak{b}$ (see [5, Theorem 3.1(d), p. 116]). Thus the above-mentioned question is nontrivial only in case $\mathfrak{b}<\operatorname{cov}(R)<\mathfrak{d}$ (it is known (see [7]) that $\operatorname{cov}(R) \leq \mathfrak{d})$.

The technology that exists in this area seems to be inadequate in answering the above-mentioned question. The aim of this paper is mainly to find new characterisations of the cardinals $\operatorname{cov}(R)$ and $\operatorname{add}(R)$ and then reformulate the 
problem in terms of these new characterisations. Such characterisations may be raised from simple combinatorial statements holding true in the presence of the continuum hypothesis $\mathbf{C H}$; e.g.,

For every family $\mathbf{D},|\mathbf{D}|<\mathfrak{c}$, of dense open sets of the real line $R$ there exists a countable dense set $G \subseteq R$ such that $|G \backslash D|<\omega$ for all $D \in \mathbf{D}$.

For every family $\mathbf{D},|\mathbf{D}|<\mathfrak{c}$, of dense open sets of the real line $R$ and for every countable dense set $Q \subseteq R$ there exists a countable infinite set $G \subseteq Q$ such that $|G \backslash D|<\omega$ for all $D \in \mathbf{D}$.

For every family $\mathbf{D},|\mathbf{D}|<\mathfrak{c}$, of dense open sets of the real line $R$ and every countable dense set $Q \subseteq R$ there exists a countable dense set $G \subseteq Q$ such that $|G \backslash D|<\omega$ for all $D \in \mathbf{D}$.

Let us recall some characterisations of $\operatorname{cov}(R)$ and $\operatorname{add}(R)$ which we will use in the sequel.

Lemma 1 ([1], Miller-Bartoszynski). $\operatorname{cov}(R)>k$ iff $\operatorname{Equal}(k)$.

Lemma 2 ([6]). $\operatorname{add}(R)>k$ iff for every family $D=\left\{D_{i}: i \in k\right\}$ of dense open sets of $R$ there exists a family $Q=\left\{Q_{n}: n \in \omega\right\}$ of dense open sets of $R$ such that for every $i \in k$ there exists $n \in \omega$ with $Q_{n} \subseteq D_{i}$.

Lemma 3 ([14], Miller-Truss). $\operatorname{add}(R)>k$ iff $\operatorname{Bounded}(k)$ and $\operatorname{cov}(R)>k$.

As a corollary to Exercise C4 from [10, p. 242], one can easily establish the following lemma.

Lemma 4. Let $(P, \leq)$ and $(Q, \leq)$ be any two countable nonatomic posets. Then there exist dense sets $P^{\prime} \subseteq P$ and $Q^{\prime} \subseteq Q$ such that $\left(P^{\prime}, \leq\right)$ and $\left(Q^{\prime}, \leq\right)$ are isomorphic as posets. (There exists a bi-injective function $H: P^{\prime} \rightarrow Q^{\prime}$ such that $H(p) \leq H(q)$ iff $p \leq q$ for all $p, q \in P^{\prime}$.)

\section{Characterisations of $\operatorname{cov}(R)$}

Theorem 1. The following are equivalent for every cardinal $k$ :

(1) $\operatorname{cov}(R)>k$.

(2) $\operatorname{Equal}(k)$.

(3) For every family $\mathbf{D},|\mathbf{D}|<k$, of dense open sets of the real line $R$ there exists a countable dense set $G \subseteq R$ such that $|G \backslash D|<\omega$ for all $D \in \mathbf{D}$.

(4) For every family $\mathbf{D},|\mathbf{D}|<k$, of dense open sets of the real line $R$ there exists a countable infinite set $G$ such that $|G \backslash D|<\omega$ for all $D \in \mathbf{D}$.

(5) $\operatorname{Equal}^{*}(k)$.

(6) $\operatorname{PS}(k)$.

(7) MA $_{k}$ (countable) (Martin's Axiom restricted to countable posets).

(8) For every family $\mathbf{D},|\mathbf{D}|<k$, of dense open sets of the real line $R$ and for every countable dense set $Q \subseteq R$ there exists a countable infinite set $G \subseteq Q$ such that $|G \backslash D|<\omega$ for all $D \in \mathbf{D}$.

(9) For every $T_{1}$ space $X$ of countable $\pi$-weight and every family $D=$ $\left\{D_{i}: i \in k\right\}$ of dense open sets in $X$ there exists a countable infinite set $G \subseteq X$ such that $\left|G \backslash D_{i}\right|<\omega$ for all $i \in k$.

Proof. (1) $\leftrightarrow(2)$ and (1) $\leftrightarrow(7)$ are well known. (1) $\leftrightarrow(2)$ is Lemma 1 , and $(1) \leftrightarrow(7)$ is a consequence of Lemma 4 .

$(1) \rightarrow(3)$ and $(3) \rightarrow(4)$ are straightforward. 
(4) $\rightarrow(5)$. Fix $F=\left\{f_{i}: i \in k\right\} \subseteq^{\omega} \omega$. For every $i \in k, D_{i}=\left\{f \in^{\omega} \omega\right.$ : $f_{i}(n)=f(n)$ for some $\left.n \in \omega\right\}$ is clearly a dense open subset of ${ }^{\omega} \omega$. Using (4), Lemma 4, and standard density arguments one can easily verify that there exists a countable infinite set $G \subseteq{ }^{\omega} \omega$ such that $|G \backslash D|<\omega$ for all $i \in k$. It is not hard to see that $G$ satisfies Equal $^{*}(k)$ for the collection $F$ as required.

$(5) \rightarrow(2)$. First we need to show

Claim. Equal ${ }^{*}(k) \rightarrow$ Weak Bounded $(k)$.

Proof. Let $F \subseteq \omega^{\omega}$ be a family of size $k$. Using the assumption choose a family $\left\{g_{n}: n \in \omega\right\}$ such that

$$
(\forall f \in F)\left(\forall^{\infty} n\right)(\exists \kappa)\left(g_{n}(\kappa)=f(\kappa)\right) .
$$

By passing to a subsequence we can assume that, for every $n, g_{n}(n), g_{n+1}(n)$, $g_{n+2}(n), \ldots$ are all equal or pairwise different.

Subclaim. For every $f \in F$ there exists $n$ and $\kappa \leq 2 n$ such that $g_{\kappa}(n)=$ $f(n)$.

Proof. Suppose not, and let $f \in F$ be such that $g_{\kappa}(n) \neq f(n)$ for $\kappa \leq 2 n$. Find $\kappa_{0}$ and a sequence $\left\{n_{\kappa}: \kappa \geq \kappa_{0}\right\}$ such that $g_{\kappa}\left(n_{\kappa}\right)=f\left(n_{\kappa}\right)$ for $\kappa \geq \kappa_{0}$. By the assumption, $\kappa>2 n_{\kappa}$. Consider terms $n_{\kappa_{0}}, n_{\kappa_{0}+1}, \ldots, n_{2 \kappa_{0}}$. Note that all these terms are smaller than $\kappa_{0}$. Thus, for some $i<j, n_{\kappa_{0}+i}=n_{\kappa_{0}+j}=n^{*}$. It follows that

$$
g_{\kappa_{0}+i}\left(n^{*}\right)=g_{\kappa_{0}+j}\left(n^{*}\right)=f\left(n^{*}\right) .
$$

In particular, $g_{n^{*}}\left(n^{*}\right)=f\left(n^{*}\right)$, a contradiction finishing the proof of the subclaim.

To finish the proof of the claim define

$$
g(n)=\max \left\{g_{\kappa}(n): \kappa \leq 2 n\right\}+1 \text { for } n \in \omega .
$$

Clearly

$$
(\forall f \in F)(\exists n)(f(n)<g(n)) .
$$

This shows that $F$ cannot be dominating as required.

To complete the proof of (5) $\rightarrow(2)$, fix $F=\left\{f_{i}: i \in k\right\} \subseteq{ }^{\omega} \omega$ and let $A=\left\{A_{j}: j \in \omega\right\} \subseteq[\omega]^{\omega}$ be a partition of $\omega$. For every $j \in \omega$ let $G_{j}=$ $\{g(j, n): n \in \omega\}$ satisfy $\operatorname{Equal}^{*}(k)$ for the collection $F_{j}=\left\{f_{i} \mid A_{j}: i \in k\right\}$. Define a function $h_{j}: \omega \rightarrow \omega$ by requiring

$$
h_{i}(j)=\min \left(\left\{m:(\forall l \geq m)\left(\exists u \in A_{j}\right)\left(g(j, l)(u)=f_{i}(u)\right)\right\}\right) .
$$

Let, by the claim, $h: \omega \rightarrow \omega$ satisfy Weak Bounded $(k)$ for the collection $H=\left\{h_{i}: i \in k\right\}$, and define a function $g: \omega \rightarrow \omega$ by letting

$$
g / A_{j}=g(j, h(j)) .
$$

Clearly $g$ satisfies $\operatorname{Equal}(k)$ for the collection $F$ as required.

$(7) \rightarrow(6)$. Fix a set $A=\left\{A_{i}: i \in k\right\} \subseteq[\omega]^{\omega}$, and let $B=\left\{b_{n}: n \in\right.$ $\omega\} \subseteq[\omega]^{\omega}$ be a $\pi$-base for $A$. Clearly $D_{i}=\left\{b \in B: b \subseteq A_{i}\right\}$ is dense in $(B, \subseteq)$ for all $i \in k$. Using (7), one can easily verify that there exists a filter $G=\left\{b_{n}: n \in \omega\right\}$ of $(B, \subseteq)$ meeting each $D_{i}$ nontrivially. Via an easy induction pick for every $n \in \omega$,

$$
s_{n} \in\left(b_{n} \backslash\left\{s_{m}: m \in n\right\}\right) .
$$

Clearly, $S=\left\{s_{n}: n \in \omega\right\}$ is an infinite pseudointersection for $A$ as required. 
$(6) \rightarrow(8)$. Let $D=\left\{D_{i}: i \in k\right\}, Q$, and $B$ be a family of dense open sets, a countable dense set, and a countable base for $R$, respectively.

Put

$$
A_{i}=D_{i} \cap Q \text { for all } i \in k
$$

and

$$
B_{b}=b \cap Q \text { for all } b \in B \text {. }
$$

Clearly $B^{*}=\left\{B_{b}: b \in B\right\}$ is a $\pi$-base for $A=\left\{A_{i}: i \in k\right\}$. Thus, by (6), there exists an infinite pseudointersection $G \subseteq Q$ for $A$. This certainly implies that $\left|G \backslash D_{i}\right|<\omega$ for all $i \in k$ as required.

$(8) \rightarrow(4)$ and $(6) \rightarrow(9)$ are straightforward.

To finish the proof of the theorem it suffices to show

$(9) \rightarrow(6)$. Fix $A=\left\{A_{i}: i \in k\right\} \subseteq[\omega]^{\omega}$, and let $B=\left\{B_{n}: n \in \omega\right\} \subseteq[\omega]^{\omega}$ be a $\pi$-base for $A$. Without loss of generality we may assume that $\bigcup A=$ $\bigcup B=\omega$. For every $x, n \in \omega$, we let $B_{n}(x)=B_{n} \backslash\{x\}$ and put

$$
F=\left\{B_{n}(x): n, x \in \omega\right\} \text {. }
$$

Clearly the topology $T_{F}$ which is produced from the subbase $F$ is $T_{1}$ and second countable. Furthermore, $A$ is a family of dense open sets in the topological space $\left(\omega, \mathbf{T}_{F}\right)$. Thus by (9) there exists a set $G \subseteq X,|G|=\omega$, such that $\left|G \backslash A_{i}\right|<\omega$ for all $i \in k$ as required.

As an easy corollary of Theorem $1(4)$ we have $\operatorname{cf}(\operatorname{cov}(R))>\omega$. In fact, more than that is true. Namely, let $\mathbf{s}_{\mathrm{c}}$ denote the least $k$ for which the following statement fails.

For every family $G \subseteq{ }^{\omega} \omega,|G|<\mathfrak{d}$, and every family $F=\left\{f_{i}: i \in k\right\} \subseteq{ }^{\omega} \omega$ there exists a family $H=\left\{h_{n}: n \in \omega\right\} \subseteq{ }^{\omega} \omega$ such that for every $g \in G$ if $g$ meets all but less than $k$ many members of $F$, then $g$ meets all but finitely many members of $H$

Then, we have:

Corollary 1. If $\operatorname{add}(R) \leq \mathbf{s}_{\mathrm{c}}$, then $\operatorname{cf}(\operatorname{cov}(R)) \geq \operatorname{add}(R)$.

Proof. Assume on the contrary, and let

$$
\operatorname{cf}(\operatorname{cov}(R))=\lambda<\operatorname{add}(R) \leq \mathbf{s}_{\mathrm{c}} .
$$

In view of [5, Theorem 3.1(d), p. 116], we may assume that $k=\operatorname{cov}(R)<\mathfrak{d}$. Fix, by Theorem 1, a family

$$
G=\left\{g_{i}: i \in k\right\} \subseteq{ }^{\omega} \omega
$$

such that

$$
\left(\forall H \in\left[{ }^{\omega} \omega\right]^{\omega}\right)(\exists i \in k)\left(\exists^{\infty} h \in H\right)\left(h \cap g_{i}=\varnothing\right) .
$$

Fix $\left\{\lambda_{j}: j \in \lambda\right\}$ a cofinal set in $k$. For every $j \in \lambda$ let $f_{j}: \omega \rightarrow \omega$ be such that

$$
\left(\forall i \in \lambda_{j}\right)\left(\exists^{\infty} n\right)\left(g_{i}(n)=f_{j}(n)\right) \text {. }
$$

Put $F=\left\{f_{j}: j \in \lambda\right\}$, and note that

$$
(\forall i \in k)\left(\left(\forall^{\infty} j \in \lambda\right)\left(f_{j} \cap g_{i} \neq \varnothing\right)\right) .
$$

Fix, by $\mathbf{s}_{\mathfrak{c}}>\lambda$, a set $H \in\left[{ }^{\omega} \omega\right]^{\omega}$ satisfying

$$
(\forall i \in k)\left(\forall^{\infty} h \in H\right)\left(h \cap g_{i} \neq \varnothing\right) \text {. }
$$

This contradicts the choice of $G$ and finishes the proof. 


\section{Characterisations of $\operatorname{add}(R)$}

Theorem 2. The following are equivalent for every cardinal $k$ :

(1) $\operatorname{add}(R)>k$.

(2) $\operatorname{SPS}(k)$.

(3) For every family $\mathbf{D},|\mathbf{D}|<k$, of dense open sets of the real line $R$ and every countable dense set $Q \subseteq R$ there exists a countable dense set $G \subseteq Q$ such that $|G \backslash D|<\omega$ for all $D \in \mathbf{D}$.

(4) $\operatorname{Equal}^{*}(k)$ and $\operatorname{Bounded}(k)$.

(5) Equal $(k)$ and $\operatorname{Bounded}(k)$.

(6) $\mathrm{MA}_{k}$ (countable) and $\operatorname{Bounded}(k)$.

(7) For every $T_{1}$ space $X$ of countable $\pi$-weight, every family $D=\left\{D_{i}\right.$ : $i \in k\}$ of dense open sets in $X$, and every countable dense set $Q \subseteq X$ there exists a dense set $G \subseteq Q$ such that $\left|G \backslash D_{i}\right|<\omega$ for all $i \in k$.

Proof. (1) $\rightarrow$ (3) follows immediately from Lemma 2.

$(3) \rightarrow(4)$. In view of Theorem 1 and Lemma 3, we only have to show that (3) implies $\operatorname{Bounded}(k)$. Fix $F=\left\{f_{i}: i \in k\right\} \subseteq{ }^{\omega} \omega$. Without loss of generality we may assume that each $f_{i}$ is a strictly increasing function. It is easy to see that

$$
\mathbf{D}_{i}=\left\{f \in{ }^{\omega} \omega: f(n)>f_{i}(n) \text { for some } n \in \omega\right\}
$$

is a dense open set of the Baire space $\omega^{\omega}$. Let

$$
G=\left\{g_{n}: n \in \omega\right\} \subseteq^{\omega} \omega,
$$

where $g_{n}$ is eventually equal to zero. Clearly $G$ is dense in $\omega^{\omega}$. By (3), Lemma 4 , and standard density arguments, there exists a dense set $Q=\left\{q_{n}\right.$ : $n \in \omega\} \subseteq G$ such that

$$
\left|Q \backslash D_{i}\right|<\omega \text { for all } i \in k .
$$

Choose a subsequence $Q^{\prime}=\left\{q_{n_{v}}: v \in \omega\right\}$ of $Q$ such that

$$
\left(\forall u \geq n_{v}\right)\left(q_{n_{v}}(u)=0\right) \wedge(\forall v \in \omega)\left(\left(q_{n_{v+1}} \mid n_{v}\right)=0\right) .
$$

On the basis of $Q^{\prime}$ we define a function $f: \omega \rightarrow \omega$ by requiring

$$
f(u)=\max \left\{q_{n_{v}}(t): v \leq u+1, t \in \operatorname{Dom}\left(q_{n_{v}}\right)\right\} .
$$

$f$ dominates $F$. Indeed, fix $i \in k$, and let $v^{\prime} \in \omega$ satisfy

$$
\left(\forall v \geq v^{\prime}\right)\left(q_{n_{v}} \in D_{i}\right) \text {. }
$$

This means that

$$
\left(\forall v \geq v^{\prime}\right)\left(\exists n \in\left[n_{v-1}, n_{v}\right)\right)\left(q_{n_{v}}(n)>f_{i}(n)\right) .
$$

Thus, if $v>v^{\prime}$, then we have

$$
f(u) \geq \max \left\{q_{n_{u+1}}(n): n \in\left[n_{u}, n_{u+1}\right)\right\}>f_{i}\left(n_{u}\right)>f_{i}(u),
$$

and the desired result follows.

Implications $(4) \rightarrow(5) \rightarrow(6) \rightarrow(1)$ are clear (Theorem 1 and Lemma 3 ).

(6) $\rightarrow(2)$. Fix $A=\left\{A_{i}: i \in k\right\} \subseteq[\omega]^{\omega}$, and let $B=\left\{b_{n}: n \in \omega\right\} \subseteq[\omega]^{\omega}$ be a base for $A$. For every $n \in \omega$, by (6), fix $S_{n}=\{s(n, m): n \in \omega\} \subseteq b_{n}$ an infinite pseudointersection for $A$. For every $i \in k$ define a function $f_{i}: \omega \rightarrow \omega$ by requiring

$$
f_{i}(n)=\min \left\{v: s(n, m) \in D_{i} \text { for all } m \geq v\right\} .
$$


Put $F=\left\{f_{i}: i \in k\right\}$. By Lemma $3(k<\operatorname{add}(R) \leq \mathfrak{b})$, there exists a function $f: \omega \rightarrow \omega$ such that

$$
(\forall i \in k)\left(\forall^{\infty} n\right)\left(f_{i}(n) \leq f(n)\right) .
$$

On the basis of $f$ we define a set $S$ by letting

$$
S=\{s(n, m): n \in \omega, m \geq f(n)\} .
$$

It can be readily verified that $S$ is a pseudointersection of $A$ meeting every member of $B$ in an infinite set as required.

$(2) \rightarrow(3)$. Fix $D=\left\{D_{i}: i \in k\right\}$ and $G$ as in (3). By Lemma 2, there is a family $Q=\left\{Q_{n}: n \in \omega\right\}$ of dense open sets of $R$ such that

$$
(\forall i \in k)(\exists n \in \omega)\left(Q_{n} \subseteq D_{i}\right) .
$$

Let $B=\left\{B_{n}: n \in \omega\right\}$ be a base for the topology of $R$. By induction, choose a set

such that

$$
T=\left\{t_{n}: n \in \omega\right\} \subseteq G
$$

$$
t_{n} \in\left(\left(B_{n} \cap\left(\bigcap\left\{Q_{m}: m \leq n\right\}\right) \cap G\right) \backslash\left\{t_{m}: m<n\right\}\right) .
$$

Clearly $T$ is dense in $R$, and $\left|T \backslash Q_{n}\right|<\omega$ for all $n \in \omega$. This, when combined with the above, finishes the proof of $(2) \rightarrow(3)$.

$(7) \rightarrow(3)$ is straightforward.

$(6) \rightarrow(7)$. It suffices in view of Theorem 1 to show that $\operatorname{PS}(k)$ and $\operatorname{Bounded}(k)$ together imply (2). This can be established as in (6) $\rightarrow(2)$ of the present theorem.

Let $\operatorname{PS}\left(\omega_{1}, k\right)$ be the generalisation of $\operatorname{PS}(k)$ to the next higher cardinal with the additional requirement that $B$ be a countable closed base $(\cap Q \in B$ for every $Q \in[B]^{\omega}$ ) of size $\omega_{1}$. Of course the continuum hypothesis must be assumed here. Also, let $\omega^{*}=\beta \omega \mid \omega$ denote the remainder of the Stone-Čech compactification of $\omega$ with the discrete topology.

Question 1. Assume CH. Does $\operatorname{PS}\left(\omega_{1}, k\right)$ imply $\operatorname{cov}\left(\omega^{*}\right)>k$ ?

Question 2. Can $\mathbf{s}_{\mathrm{c}}$ be strictly less than $\operatorname{add}(R)$ ?

\section{ACKNOWLEDGMENTS}

The author wishes to thank Professor D. H. Fremlin for all his communications and the referee for some valuable remarks concerning the notions of $\operatorname{cov}(R)$ and $\operatorname{add}(R)$ and for shaping the paper in its present form.

\section{REFERENCES}

1. T. Bartoszynski, Additivity of measure implies additivity of category, Trans. Amer. Math. Soc. 81 (1984), 209-213.

2. __ Combinatorial aspects of measure and category, Fund. Math. 127 (1987), 225-239.

3. T. Bartoszynski and J. I. Ihoda, On the cofinality of the smallest covering of the real line by meagre sets, J. Symbolic Logic 54 (1989), 828-832.

4. T. Bartoszynski, J. I. Ihoda, and S. Shelah, The cofinality of cardinal invariants related to measure and category, J. Symbolic Logic 54 (1989), 719-726. 
5. E. K. van Douwen, The integers and topology, Handbook of Set Theoretic Topology (K. Kunen and J. E. Vaughan, eds.), North-Holland, Amsterdam, 1984, pp. 113-167.

6. D. H. Fremlin, The partially ordered sets of measure and category and Tukey's ordering, preprint.

7. _ Cichon's diagram, Seminaire d'Initiation a l'Analyse (G. Choque, M. Rogalski, and J. Saint Raymond, eds.), 23eme anne: 1983-1984, Université Pierre et Marie Curie, (Paris-VI), Paris, expose 5.

8. T. Jech, Set theory, Academic Press, New York, 1978.

9. K. Keremedis, Partition reals and the consistency of $t<\operatorname{add}(R)$, Math. Log. Quart. 39 (1993).

10. K. Kunen, Set theory, North-Holland, Amsterdam, 1983.

11. _ Random and Cohen reals, Handbook of Set Theoretic Topology (K. Kunen and J. E. Vaughan, eds.), North-Holland, Amsterdam, 1984, pp. 887-911.

12. A. W. Miller, $A$ characterisation of the least cardinal number for which the Baire category theorem fails, Proc. Amer. Math. Soc. 86 (1982), 498-502.

13. _ The Baire category theorem and cardinals of countable cofinality, J. Symbolic Logic 47 (1982), 275-288.

14. $\longrightarrow$ Some properties of measure and category, Trans. Amer. Math. Soc. 266 (1981), 93-114.

15. J. E.Vaughan, Small uncountable cardinals and topology, Open Problems in Topology (J. van Mill and G. M. Reed, eds.), North-Holland, Amsterdam, 1990.

Department of Mathematics, University of Aegean, Karlovasi 83200, Samos, Greece

E-mail address: kker@pythagoras. aegean. ariadne-t.gr. 